\title{
Bacterial inactivation/sterilization by argon plasma treatment on contaminated titanium implant surfaces: In vitro study
}

\author{
Marco Annunziata ${ }^{1 *}$, Luigi Canullo ${ }^{2,3^{*}}$, Giovanna Donnarumma ${ }^{4}$, Pina Caputo ${ }^{4}$, Livia Nastri ${ }^{1}$, Luigi Guida ${ }^{1}$
}

\author{
${ }^{1}$ Multidisciplinary Department of Medical-Surgical and Dental Specialties, Second University of Naples, Naples, Italy \\ ${ }^{2}$ Istituto Stomatologico Toscano, Viareggio, Italy \\ ${ }^{3}$ Private Practice in Rome, Rome, Italy \\ ${ }^{4}$ Department of Experimental Medicine, Microbiology Section, Second University of Naples, Naples, Italy
}

Correspondence:

Via Nizza, 46 - 00198

Rome-Italy

lugicanullo@yahoo.com

Received: 25/05/2015 Accepted: 05/09/2015

\begin{abstract}
Annunziata M, Canullo L, Donnarumma G, Caputo P, Nastri L, Guida L. Bacterial inactivation/sterilization by argon plasma treatment on contaminated titanium implant surfaces: In vitro study. Med Oral Patol Oral Cir Bucal. 2016 Jan 1;21 (1):e118-21.

http://www.medicinaoral.com/medoralfree01/v21i1/medoralv21i1p118.pdf
\end{abstract}

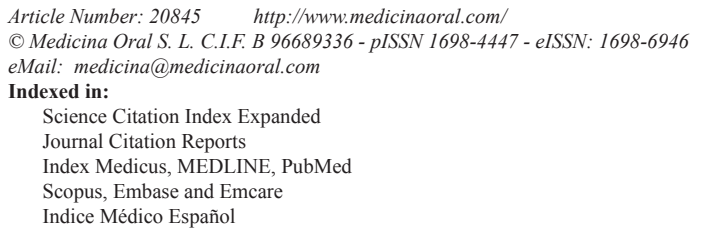

\begin{abstract}
Background: Surface treatment by argon plasma is widely used as the last step of the manufacturing process of titanium implant fixtures before their sterilization by gamma rays. The possibility of using such a technology in the daily clinical practice is particularly fascinating. The aim of the present study was to assess the effects of the argon plasma treatment on different titanium implant surfaces previously exposed in vitro to bacterial contamination. Material and Methods: Sterile c.p. titanium implant discs with turned (T, Sa: $0.8 \mu \mathrm{m})$, sandblasted/acid-etched (SAE, Sa: $1.3 \mu \mathrm{m}$ ) and titanium plasma sprayed (TPS, Sa: $3.0 \mu \mathrm{m}$ ) surface were used in this study. A strain of Aggregatibacter actinomycetemcomitans ATCC 3718 was grown at $37^{\circ} \mathrm{C}$ under anaerobic conditions for $24 \mathrm{~h}$ and then transferred on six discs for each of the three surface types. After 24 hours, a half of the contaminated discs (control group) were directly used to evaluate the colony forming units (CFUs). The other half of the contaminated discs (test group) were treated in an argon plasma chamber for 12 minutes at room temperature prior to be analyzed for CFU counting. All assays were performed using triplicate samples of each material in 3 different experiments.

Results: When the CFU counting was carried out on control discs, a total of $1.50 \times 10^{6} \pm 1.4 \times 10^{5}, 1.55 \times 10^{6} \pm 7.07 \times 10^{4}$ and $3.15 \times 10^{6} \pm 2.12 \times 10^{5} \mathrm{CFU}$ was respectively assessed for T, SAE and TPS discs, without statistically significant differences among the three surfaces. On the contrary, any trace of bacterial contamination was assessed for titanium discs treated in the argon plasma chamber prior to be analyzed, irrespectively to the implant surface tested.

Conclusions: Within the limit of this study, reported data suggested that the argon plasma technology could be efficiently used to decontaminate/sterilize previously infected titanium implant surfaces.
\end{abstract}

Key words: Argon plasma, titanium implant surface, Aggregatibacter actinomycetemcomitans. 


\section{Introduction}

Surface treatment by argon plasma is widely used as the last step of the manufacturing process of titanium implant fixtures before their sterilization by gamma rays. This technology works through the activation of the electronic mantle of materials by a spray of argon under pressure at room temperature. The main microscopic effect of such activation is the removal of the microbiologic pollution and contamination from the metallic surfaces. At the same time, this process is also able to modify the physico-chemical, and in turn the biological features of implant surfaces, and their interaction with the environment (1-4).

The possibility of using such a technology outside the industrial field in the daily clinical practice is particularly fascinating. In particular, the activation of prosthetic titanium implant components before their installation in the oral cavity could represent a very advantageous field of application of the argon plasma treatment. Recent studies, in this sense, have suggested that treatment of titanium abutments by argon plasma may enhance peri-implant soft tissue healing at an early stage. I was associated with the preservation of the marginal bone level over time $(5,6)$.

Another promising field of application of the argon plasma technology could be the therapy of peri-implant disease, through the treatment of contaminated prosthetic components and implant surfaces, once a specific handpiece for chair-side use will be available.

However, so far, the effectiveness of plasma technology to decontaminate/sterilize previously infected titanium implant surfaces has not been proven, neither the effect of different surface textures on such decontamination. The aim of the present study was to assess the effects of the argon plasma treatment on different titanium implant surfaces (turned, sand-blasted/acid-etched and titanium plasma sprayed) previously exposed in vitro to bacterial contamination.

\section{Material and Methods}

Sterile c.p. titanium implant discs $(5 \mathrm{~mm}$ wide, $3 \mathrm{~mm}$ height) with turned (T), sandblasted/acid-etched (SAE) and titanium plasma sprayed (TPS) surface (Sweden \& Martina, Padua, Italy) were used in this study. The average roughness $(\mathrm{Sa})$ of these surfaces was $0.8 \mu \mathrm{m}, 1.3$ $\mu \mathrm{m}$ and $3.0 \mu \mathrm{m}$ for T, SAE and TPS discs, respectively. A strain of Aggregatibacter actinomycetemcomitans (Aa) ATCC3718 was grown at $37^{\circ} \mathrm{C}$ under anaerobic conditions for $24 \mathrm{~h} .2 \times 10^{6}$ bacteria cells were inoculated into 48-well flat-bottomed sterile polystyrene microplates (Costar; Corning, Inc., NY, USA) in which were inserted six discs for each of the three surface types and incubated for $24 \mathrm{~h}$ at $37^{\circ} \mathrm{C}$. To enhance biofilm formation, discs were previously coated for 1 hour with human saliva. An unstimulated saliva sample was ob- tained from a healthy male donor, who had not assumed any medication for 3 months prior to the study. The protocol was approved by the Ethics Committee and a specific informed consent was signed by participants. After 24 hours, a half of the contaminated discs (control group) were washed three times with PBS to remove the planktonic and loosely attached bacteria and transferred to a $15 \mathrm{ml}$ Falcon tube containing $1 \mathrm{ml}$ of PBS. The tubes were sonicated for $1 \mathrm{~min}$ at $100 \%$ intensity for the disruption of the biofilms. Sonication fluid was diluted and all serial dilutions were then used to evaluate the colony forming units (CFUs). The other half of the contaminated discs (test group) were inserted on a metallic holder and treated in an argon plasma chamber (Plasma $\mathrm{R}$, Sweden \& Martina, Padua, Italy) for 12 minutes at room temperature (Fig. 1) prior to be washed, sonicated and analyzed for CFU counting as above mentioned. All assays were performed using triplicate samples of each material in 3 different experiments.

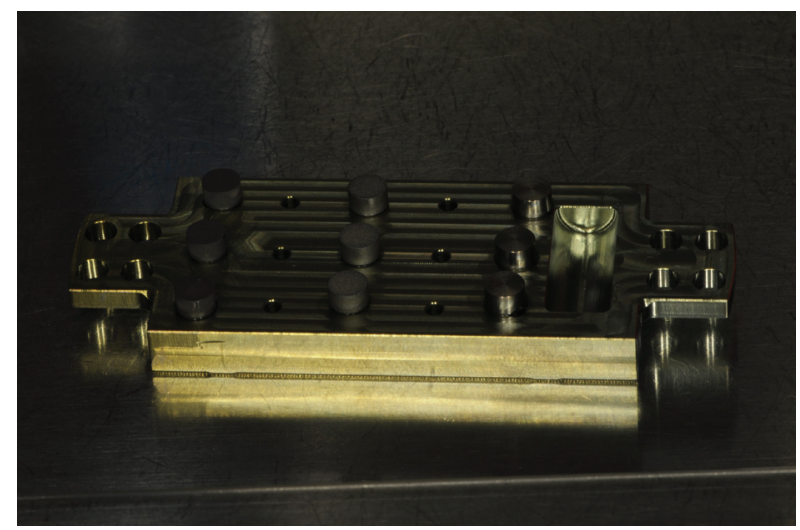

Fig. 1. Titanium discs with turned, sandblasted/acid-etched and titanium plasma sprayed surface inserted on a metallic holder and treated in the argon plasma chamber.

\section{Results}

When the CFU counting was carried out on control discs, a total of $1.50 \times 10^{6} \pm 1.4 \times 10^{5}, 1.55 \times 10^{6} \pm 7.07 \times 10^{4}$ and $3.15 \times 10^{6} \pm 2.12 \times 10^{5}$ CFU was respectively assessed for $\mathrm{T}$, SAE and TPS discs, without statistically significant differences among the three surfaces. On the contrary, any trace of bacterial contamination was found for the titanium discs treated in the argon plasma chamber prior to be analyzed, irrespectively to the implant surface tested (Fig. 2).

\section{Discussion}

Data from the present in vitro study demonstrated that plasma of argon is suitable for removal of any trace of bacterial contamination on previously contaminated titanium discs with different micro-topography.

Aggregatibacter actinomicetemcomitans was used as a test microorganism because of its presence in the human 


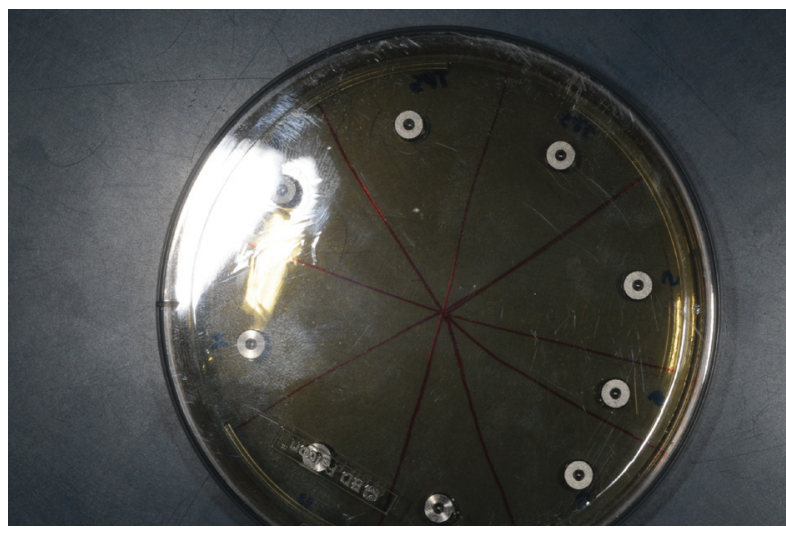

Fig. 2. Any trace of bacterial contamination was found on the titanium discs treated in the argon plasma chamber, irrespectively to the implant surface tested.

oral cavity and in the subgingival biofilm. This microorganism, indeed, is a Gram-negative facultative nonmotile rod often found in association with periodontitis, especially in its more aggressive forms (7). At the same time, A. actinomicetemcomitans has been shown also to have a role in the peri-implant disease, together with gram-negative anaerobic bacteria commonly associated to chronic periodontitis (Porphyromonas gingivalis, Treponema denticola, Tannerella forsythia, Fusobacterium sp., Prevotella intermedia) and micro-organisms associated to therapy-resistant (refractory) periodontitis (Staphylococcus aureus, Candida Albicans) (8).

In the present study, the microbiologic analysis of the titanium disks previously contaminated by Aa for $24 \mathrm{~h}$ and not treated by the argon plasma chamber (control group), demonstrated bacterial adhesion to all surface types: turned, sandblasted/acid-etched and titanium plasma sprayed. In particular, a trend for slightly higher bacterial adhesion on rougher surfaces was observed, but no statistical significance was detected. This finding was not in accordance with the main part of the literature, in which a direct correlation between bacterial adhesion and surface roughness of titanium implants is generally reported (9). However, it is known how such correlation is based on largely descriptive studies and numerous variables may potentially affect the obtained results and make difficult to compare different studies, including the specific surface treatments applied and the absence of a universally accepted classification system for implant surface features.

According to the data reported, a 12 minute-cycle in the argon plasma chamber allowed to eliminate any trace of bacteria from the three surfaces adopted in the study, irrespectively of their different surface roughness. This is in accordance with $\mathrm{Yu}$ et al. (10) who demonstrated that the argon plasma is effective in the inactivation/ sterilization of surfaces from oral bacteria, and Young- blood \& Ong (11), who suggested the use of plasmaglow discharge as an alternative sterilization procedure for medical and dental implants.

Actually, the term "sterilization" refers to any process that eliminates/kills all micro-organisms, including bacteria, spores, fungi, viruses and prions. Although few data are available about their effect on prions, which still remain the hardest challenge for any medical sterilization procedure, several studies have shown the ability of cold atmospheric plasmas to promote the decontamination of inanimate objects, thanks to their high antimicrobial efficacy and their easy access into narrow and confined spaces $(12,13)$.

The possible mechanism underlying the antibacterial effect of the non-thermal low temperature atmospheric argon plasma has been extensively studied over the past decade (14). It was concluded that effective inactivation of micro-organisms is based on plasma-generated highly reactive agents including UV photons, oxygen species, charged particles as well as electric fields (15).

The main limitation of the present study was represented by the fact that only one bacterial species was used to contaminate disks. Together with the short time of incubation, this did not allow the formation of a well structured biofilm, and further in vitro and ex vivo studies are needed to confirm the encouraging, although preliminary, obtained results.

\section{Conclusions}

Within the limit of this study, reported data suggested that the argon plasma technology could be efficiently used to decontaminate/sterilize previously infected titanium implant surfaces. Such encouraging results potentially widen the use of this technology also for the decontamination of infected rough implant surfaces, with the aim to positively affect the response of soft and hard peri-implant tissues. It might open to new possible strategies in the field of peri-implantitis therapy once a specific handpiece for chair-side use would be available.

\section{References}

1. Aronsson BO, Lausmaa J, Kasemo B. Glow discharge plasma treatment for surface cleaning and modification of metallic biomaterials. J Biomed Mater Res. 1997;35:49-73.

2. Coelho PG, Giro G, Teixeira HS, Marin C, Witek L, Thompson VP, et al. Argon-based atmospheric pressure plasma enhances early bone response to rough titanium surfaces. J Biomed Mater Res A. 2012;100:1901-6.

3. Giro G, Tovar N, Witek L, Marin C, Silva NR, Bonfante EA, et al. Osseointegration assessment of chairside argon-based nonthermal plasma-treated Ca-P coated dental implants. J Biomed Mater Res A. 2013;101:98-103.

4. Teixeira HS, Marin C, Witek L, Freitas A Jr, Silva NR, Lilin T, et al. Assessment of a chair-side argon-based non-thermal plasma treatment on the surface characteristics and integration of dental implants with textured surfaces. J Mech Behav Biomed Mater. 2012;9:45-9. 
5. Canullo L, Peñarrocha-Oltra D, Marchionni S, Bagán L, Peñarrocha-Diago MA, Micarelli C. Soft tissue cell adhesion to titanium abutments after different cleaning procedures: preliminary results of a randomized clinical trial. Med Oral Patol Oral Cir Bucal. 2014;19:177-83.

6. Canullo L, Peñarrocha D, Clementini M, Iannello G, Micarelli C. Impact of plasma of argon cleaning treatment on implant abutments in patients with a history of periodontal disease and thin biotype: radiographic results at 24-month follow-up of a RCT. Clin Oral Implants Res. 2015;26:8-14.

7. Könönen E, Müller HP. Microbiology of aggressive periodontitis. Periodontol 2000. 2014;65:46-78.

8. Mombelli A, Decaillet F. The characteristics of biofilms in periimplant disease. J Clin Periodontol 2011;38 (Suppl. 11):203-13.

9. Subramani K, Jung RE, Molenberg A, Hammerle CH. Biofilm on dental implants: a review of the literature. Int J Oral Maxillofac Implants. 2009;24:616-26.

10. Yu QS, Huang C, Hsieh FH, HuffH, Duan Y. Bacterial inactivation using a low-temperature atmospheric plasma brush sustained with argon gas. J Biomed Mater Res B Appl Biomater. 2007;80:211-9.

11. Youngblood T, Ong JL. Effect of plasma-glow discharge as a sterilization of titanium surfaces. Implant Dent. 2003;12:54-60.

12. Isbary G, Shimizu T, Li YF, Stolz W, Thomas HM, Morfill GE, et al. Cold atmospheric plasma devices for medical issues. Expert Rev Med Devices. 2013;10:367-77.

13. Mai-Prochnow A, Murphy AB, McLean KM, Kong MG, Ostrikov KK. Atmospheric pressure plasmas: infection control and bacterial responses. Int J Antimicrob Agents. 2014;43:508-17.

14. Lackmann JW, Bandow JE. Inactivation of microbes and macromolecules by atmospheric-pressure plasma jets. Appl Microbiol Biotechnol. 2014;98:6205-13.

15. Moreau M, Orange N. Feuilloley MGJ (2008) Non-thermal plasma technologies: New tools for bio-decontamination. Biotechnol Adv. 26:610-7.

*C.L and A.M. contributed equally to this work 